\title{
Plasmonic induced transparency in a coupled system composed of metal-insulate-metal stub and trapezoid cavity resonator
}

\author{
Pengfei Zheng, Huimin Yang, Linsen Jiao, Meiyong Fan, Binfeng Yun*, Yiping Cui* \\ Advanced Photonics Center, Southeast University, Nanjing, China, 210096
}

\begin{abstract}
A plasmonic induced transparency system constructed by a metal-insulate-metal stub coupled with a trapezoid cavity resonator was proposed. The results show that the spectra of different narrow modes in the trapezoid resonator can overlap with the broad stub mode and induce the plasmonic induced transparency effect. However, some of them cannot produce a plasmonic induced transparency effect because there is hardly any near field overlap between the trapezoid cavity mode and the stub mode, which was proved by the mode field distributions in the coupled resonator system. The "disappeared" plasmonic induced transparency can be reproduced by changing the relative position between the stub and trapezoid resonator. Also the coupling strength can be modulated by this method to manipulate the plasmonic induced transparency and slow light effect.
\end{abstract}

Keywords: Surface plasmon polaritons, plasmonic induced transparency, trapezoid cavity, slow-light

\section{Introduction}

Surface plasmon polaritons (SPPs) are the electromagnetic waves coupled with free-electrons in metal and trapped on the interface between metal and dielectric materials. The SPP waveguide based on metal-insulator-metal (MIM) structure has attracted much attention because electromagnetic waves can be restricted in subwavelength scale that makes MIM plasmonic structure possible for integrated photonic devices.

Electromagnetically induced transparency (EIT) is a quantum effect occurred in an atomic system, where a quantum destructive interference between two different excitation pathways leads to a transparency window with strong dispersion and a significant slow light effect near the transparency peak. Also it has been proved to have promising applications in nonlinear optical processing, ultrafast switching and optical data storage for its noble property of slow light effect. An EIT-like effect, also known as coupled resonance induced transparency (CRIT) that occurs in some coupled micro-cavities has been analyzed theoretically and observed experimentally in normal silicon based photonics systems [1-3]. Besides, the EIT-like effect can also be found in plasmonic systems based on the MIM structures. Until now, a lot of works have been done to analyze the EIT-like phenomenon based on coupled plasmonic resonators which are also known as plasmonic induced transparency (PIT). Lu et al. theoretically investigated the PIT effect in a plasmonic system composed of multiple cascaded micro-disk resonators which were aperture-side-coupled to MIM bus waveguides [4]. Based on the disk resonator, a serious of PIT systems have been investigated [5, 6]. Moreover, there are also some articles refer to PIT systems based on ring resonators [7] and F-P resonators [8-10]. Some studies have focused on rectangular resonators which can provide unique properties by their novel designs [11-13]. On the basis of

\footnotetext{
* Corresponding author. Tel: +86 25 83792470-8208; fax: +86 2583790201

Email addresses: ybf@seu.edu.cn; cyp@seu.edu.cn .
} 
these designs, some new structures were proposed to implement more functionality by modulating the offset positions of resonators [14] or rotating the angles of the ring resonators [15]. Recently, Song et al. proposed a trapezoid resonator which shows novel transmission properties and mode distributions_[16]. In Song's paper, they discussed the dependence of transmission spectra and resonance wavelengths on the parameters of the trapezoid resonator.

It is well known that the near field coupling of two MIM plasmonic resonators can induce the PIT phenomenon. Here, a PIT system based on an MIM stub resonator coupled with a trapezoid resonator was proposed to realize the PIT and slow light effect. To analyze this system conveniently, the modes of trapezoid resonator were defined and their variation trends depended on the lower bottom length of trapezoid were analyzed. Then the PIT effects induced by different modes of trapezoid resonator coupled with stub were analyzed and some abnormal phenomena were found. Some of the mode-coupling systems cannot generate a PIT effect, although the wavelengths of trapezoid mode and the stub are well overlapped. By investigating the mode distributions of those PIT systems, we found the mode overlap between those trapezoid modes and stub modes is too weak that causes a very weak coupling strength between the two resonators and leads to the absence of the PIT effect. Further investigation shows that the relative position between the two resonators affects the overlap of their mode fields, which can determine the coupling strength and the slow light effect based on the proposed PIT system. By altering the relative position, the PIT and slow light effect can be modulated and optimized to achieve the largest group index.

All the results in this article were simulated by the finite-difference time-domain (FDTD) method with grid size of $1 \mathrm{~nm} \times 1 \mathrm{~nm}$. Our work offers a new perspective through the PIT based slow light device and we expect that our work would do some help to the design of other PIT systems and make the slow light system more efficiently. The plasmonic devices can be fabricated by a sequence of lithography and etch processes. A silver film will be evaporated onto a substrate which can be SOI wafer or glass. Then, the designed profile structure will be etched by electron beam lithography and etch process. Fiber to chip couplers should be fabricated by lithography step and dry etching at the two ports of the plasmonic device [17, 18].

\section{Resonance modes in trapezoid resonator}

The structure of trapezoid resonance system is shown in Fig.1, where $L_{1}, L_{2}, H$ and $g$ are the upper bottom length, the lower bottom length, the height of the trapezoid cavity and the gap between the trapezoid cavity and the bus waveguide, respectively. The width of bus waveguide, noted as $w$, is fixed as $50 \mathrm{~nm}$.

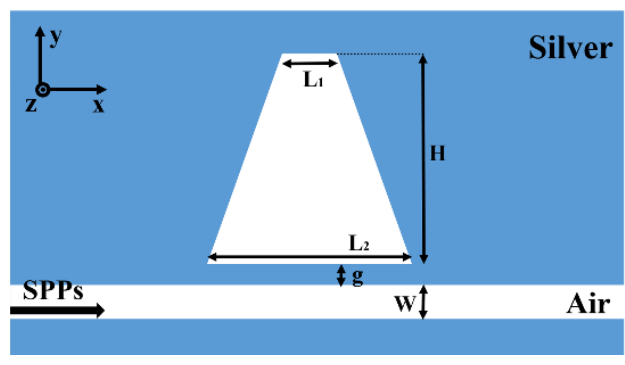

Fig.1. Schematic of trapezoid resonator coupled with MIM bus waveguide directly.

The basic theory to analyze this system is coupled mode theory (CMT), with which we can get the transmission property theoretically $[4,5]$. In this system, the permittivity of silver is given by 
the Drude model: $\varepsilon(\omega)=\varepsilon_{\infty}-\omega_{p}{ }^{2} \mu\left(\omega^{2}+i \omega \gamma\right)$, where $\underline{\varepsilon}_{\infty}$ is the relative permittivity at infinite frequency with the value of $3.7, \omega_{p}$ is the plasma frequency which is set as $1.38 \times 10^{16} \mathrm{~Hz}, \gamma$ is the damping frequency of the oscillations and the value is $2.73 \times 10^{13} \mathrm{~Hz}$, and $\omega$ is the angular frequency of the incident light [19].

The transmission spectrum and resonance modes at different wavelengths were investigated with the parameters set as: $L_{1}=50 \mathrm{~nm}, L_{2}=250 \mathrm{~nm}, H=300 \mathrm{~nm}$ and $g=20 \mathrm{~nm}$. Fig. 2 (a) shows the transmission spectrum, in which three resonant modes can be observed. As only TM modes are supported by the MIM plasmonic waveguides, the modes are defined as $\mathrm{TM}_{\mathrm{mn}}$, where $\mathrm{m}$ and $\mathrm{n}$ denote the number of horizontal and vertical zero points in the mode fields of trapezoid resonator, respectively. The similar definitions were proposed in Ref. [12].
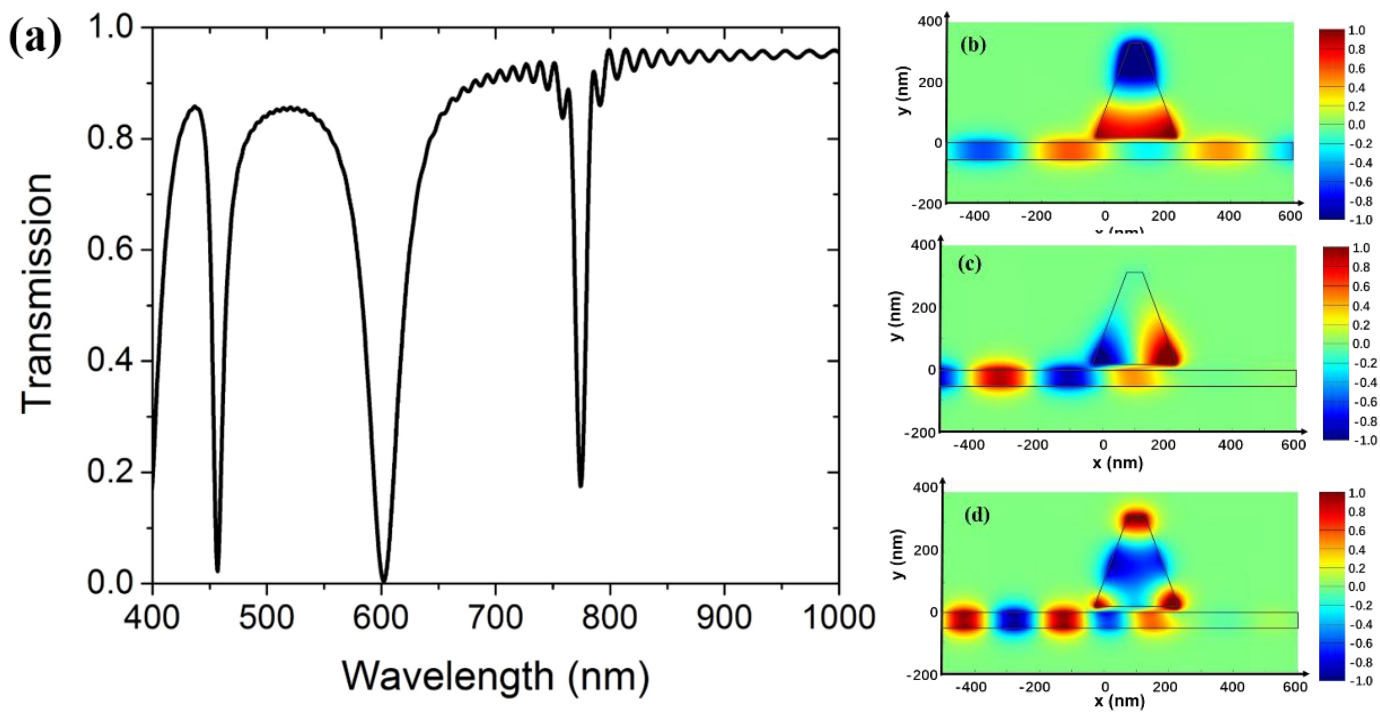

Fig.2. (a) The transmission spectrum of trapezoid resonator coupled with MIM waveguide, with parameters of trapezoid set as $L_{l}=50 \mathrm{~nm}, L_{2}=250 \mathrm{~nm}, H_{l}=300 \mathrm{~nm}, g=20 \mathrm{~nm}$; (b)-(d) Normalized $\left|H_{z}\right|$ fields of $\mathrm{TM}_{01}, \mathrm{TM}_{10}$ and $\mathrm{TM}_{12}$ modes at the wavelengths of $776.69 \mathrm{~nm}, 613.11 \mathrm{~nm}$ and $456.13 \mathrm{~nm}$.

The influences of lower bottom lengths $\left(L_{2}\right)$ on the transmission spectra and relevant modes were investigated. Fig. 3(a) shows the transmission spectra of the trapezoid resonator versus different lower bottom length $L_{2}$. With the increasing of $L_{2}$, the variation trends of the resonance wavelengths of different modes are complex. The resonance wavelength of $\mathrm{TM}_{01}$ mode decreases firstly and then increases, while the resonance wavelength of $\mathrm{TM}_{10}$ increases almost linearly. As $\mathrm{TM}_{10}$ mode resonates in horizontal direction like a standing wave, whose resonance wavelength depends on horizontal length of the resonator, namely, the lower bottom length of trapezoid cavity. And $\mathrm{TM}_{01}$ mode resonates in vertical direction, so the resonance wavelength depends mainly on the height of the trapezoid cavity. As $H$ is fixed as $300 \mathrm{~nm}$, the resonance wavelength of $\mathrm{TM}_{01}$ mode is varying around the same value and is influenced by the changing of $L_{2}$. For $\mathrm{TM}_{01}$ mode, the trapezoid resonator can be treat as an F-P resonator, whose resonance wavelength is given by: $m \lambda_{\text {res }}=2 N_{\text {eff }} H$, where $m$ is an integer, $\lambda_{\text {res }}$ is the resonance wavelength, $N_{\text {eff }}$ is the effective index of the cavity. $N_{\text {eff }}$ is determined by the width of the MIM structure, namely, the lower bottom length $L_{2}$ of the trapezoid cavity as discussed in ref. [20]. With the increasing of $L_{2}$, the effective index diminished, which leads to the blue shift of resonance wavelength. Furthermore, the degeneracy of $\mathrm{TM}_{10}$ mode and $\mathrm{TM}_{12}$ mode were found when $L_{2}=150 \mathrm{~nm}$, and when $L_{2}=350 \mathrm{~nm}$, the $\mathrm{TM}_{01}$ mode 
and $\mathrm{TM}_{10}$ mode are degenerated. The resonance wavelengths of different modes versus the lower bottom length of trapezoid were extracted and plotted in Fig. 3(b). The resonance wavelength of $\mathrm{TM}_{12}$ decreases firstly and then increases with the increasing of $\mathrm{L}_{2}$, while the wavelength of $\mathrm{TM}_{30}$ increase steadily.
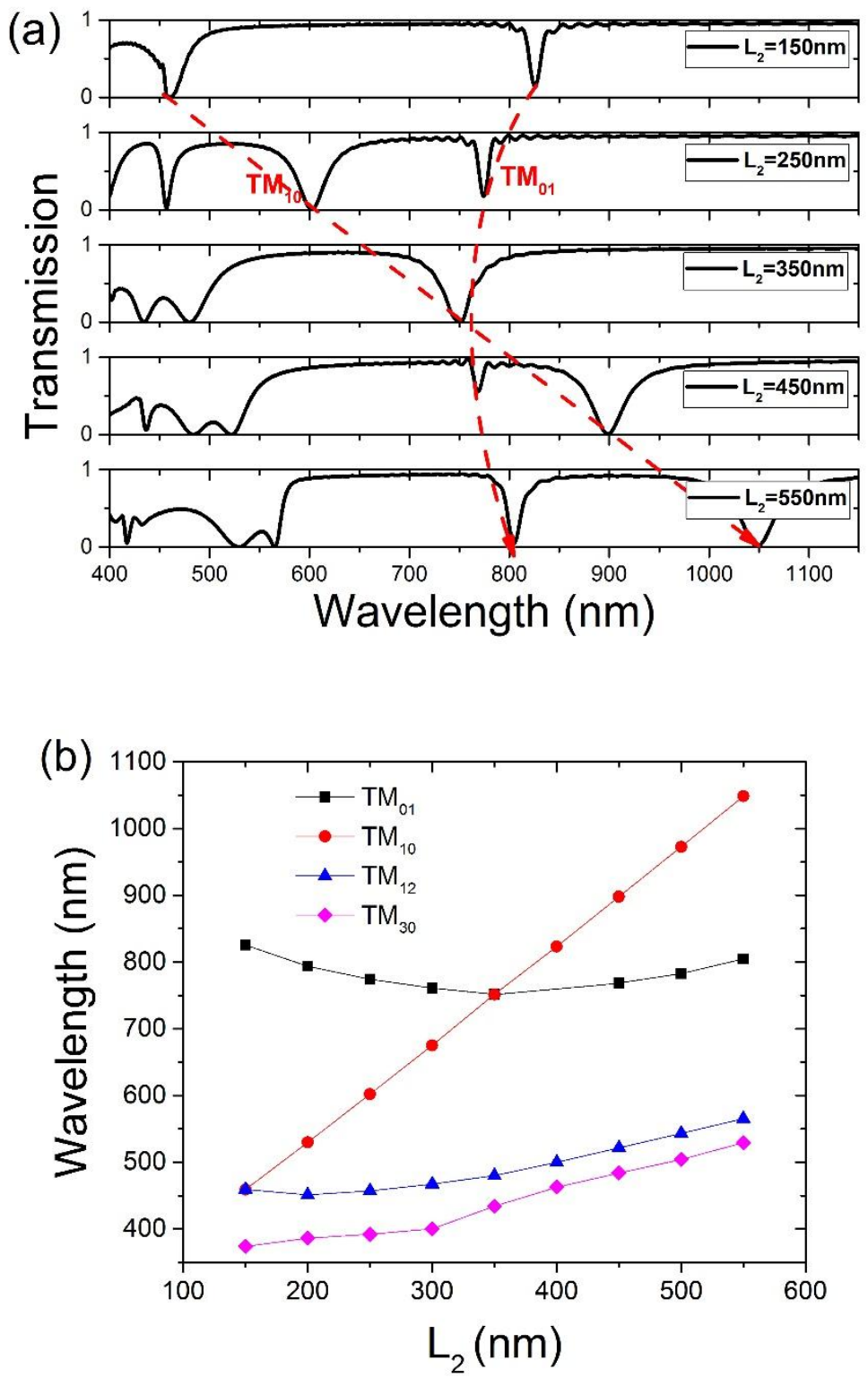

Fig.3. (a) The transmission spectra of trapezoid resonator vary with the lower bottom length. (b) The resonant wavelengths of different resonance modes vary with the lower bottom length.

\section{PIT induced by $\mathbf{T M}_{01}$ mode}

The results aforementioned indicate that many modes can resonate in the trapezoid resonator and their resonance wavelengths can be changed by altering the geometric parameters such as the lower bottom length. As known, when the resonance modes of two resonators overlap with each other, a PIT effect can be induced due to the interference of these two modes.

Stub resonator is a kind of classical MIM filer. Here, a PIT system consist of the stub resonator 
and the trapezoid resonator was proposed and shown in Fig. 4. By the near field coupling between them, a PIT effect can be achieved. The width and height of the stub are set as $W_{s}=50 \mathrm{~nm}$ and $H_{s}$ $=120 \mathrm{~nm}$, respectively. And the resonance wavelength of the stub is around $820 \mathrm{~nm}$ which is near the resonance wavelength of $\mathrm{TM}_{01}$ mode of the trapezoid.

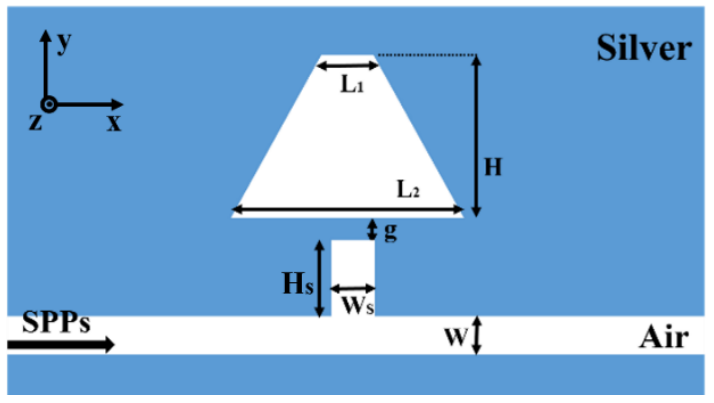

Fig.4 The structure of PIT system composed of trapezoid and stub resonators.

The PIT effect induced by two coupled resonance cavities can be analyzed theoretically by the CMT, in which the transmission is derived as [5]:

$$
T=\left|\frac{\mathrm{j}\left(\omega-\omega_{s}\right)+\gamma+\frac{\gamma}{\mathrm{j}\left(\omega-\omega_{T}\right)}}{\left(\omega-\omega_{s}\right)+\gamma+\beta+\frac{\gamma}{\mathrm{j}\left(\omega-\omega_{T}\right)}}\right|^{2}
$$

Here, $\omega_{s}$ and $\omega_{T}$ stand for the resonance frequency of stub resonator and trapezoid resonator, respectively. $\beta$ is the coupling coefficient of the waveguide and stub resonator, $\gamma$ is the coupling coefficient of the stub resonator and trapezoid micro-cavity, $j$ stands for the imaginary unit. The transmission spectrum of the PIT system with the lower bottom length of trapezoid $L_{2}=260 \mathrm{~nm}$ is shown in Fig. 5(a). The PIT peak appears at the resonance wavelength of $772.24 \mathrm{~nm}$, which is near the resonance wavelength of trapezoid resonator, with the transmission of 0.53. Fig. 5(b) is the normalized distribution of $\mathrm{z}$ component of magnetic field $\left(\mathrm{H}_{\mathrm{z}}\right)$ at the PIT wavelength, while Fig.5(c) and Fig. 5(d) are the $\mathrm{H}_{\mathrm{z}}$ distributions of the left dip $(760.93 \mathrm{~nm})$ and the right dip $(860.98 \mathrm{~nm})$, respectively. The mode field in the stub and bus waveguide have opposite phases, which leads to the destructive interference at the wavelengths of these two dips.

It is noted that the spectra in Fig.5 (a) are similar to the spectra generated by Fano resonance that gives rise to asymmetric spectra. There is no essential difference between the spectra of our PIT system and the spectra induced by Fano resonance. The PIT effect in this paper is obtained by the interaction of the stub mode and the trapezoid mode. As the Q-factor of stub is small, its spectrum is much broader than the trapezoid resonator whose Q-factor is larger. So they can also be treat as "broad bright mode" and "narrow dark mode", respectively. The generation mechanism of PIT effect is the same as Fano resonance [21, 22]. And the transparent peaks essentially come from the destructive inference of two optical paths in the plasmonic systems. 

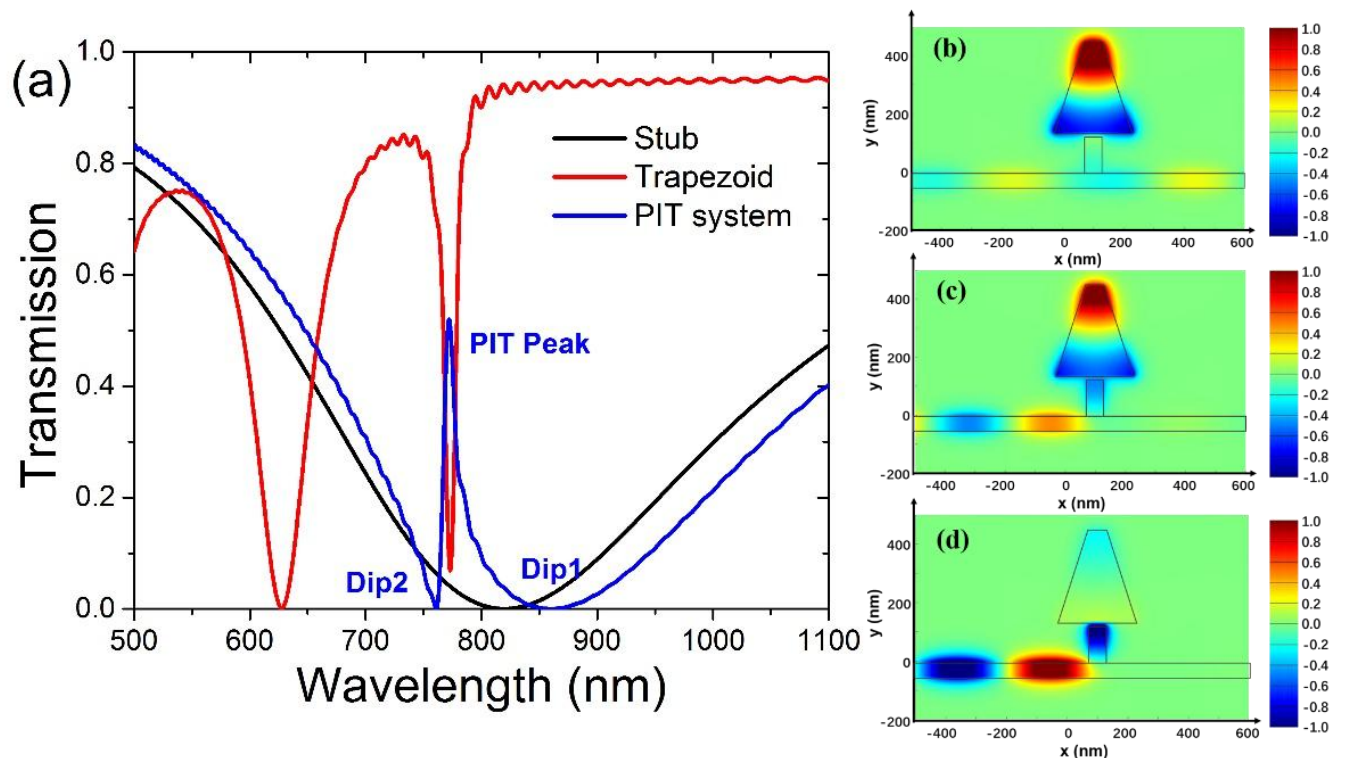

Fig. 5. (a) Transmission spectra of the stub resonator, the trapezoid resonator and the PIT system with $L_{2}=260 \mathrm{~nm}$.

(b)-(d) The $\mathrm{H}_{\mathrm{z}}$ distributions of this system at the wavelengths of PIT peak, dip1 and dip2.

Fig. 6(a) shows the transmission spectra of this system with the length of lower bottom $L_{2}$ varying from $140 \mathrm{~nm}$ to $300 \mathrm{~nm}$ with an increment of $40 \mathrm{~nm}$. The PIT wavelengths have a blue shift when $L_{2}$ increases. It is owing to the changed resonance wavelengths of trapezoid cavity by altering $L_{2}$ as discussed in the last part. According to CMT, the coupling coefficient between the trapezoid cavity and the stub is directly proportional to their mode fields overlap. When $L_{2}$ increases, the effective index of the trapezoid resonator becomes smaller and the mode field inside the cavity is weaker. That results in the decrease of the coupling coefficient between the stub and the trapezoid resonator. And according to Eq. (1), as the coupling coefficient becomes smaller, the transmission of PIT peak decreases. The transmission spectra dependence on the heights of stub are shown in Fig. 6 (b). The PIT peaks appear near the same wavelength, because the PIT wavelength is determined by the resonance wavelength of the trapezoid resonator which is unchanged here. When the stub height $\left(H_{s}\right)$ is about $110 \mathrm{~nm}$, the line-shape of transmission spectrum is symmetric because the resonance wavelength of the stub matches the trapezoid cavity's resonance wavelength relative well. Otherwise when the resonance wavelengths of the two resonators are misaligned, an PIT peak with asymmetric line-shape can be obtained.
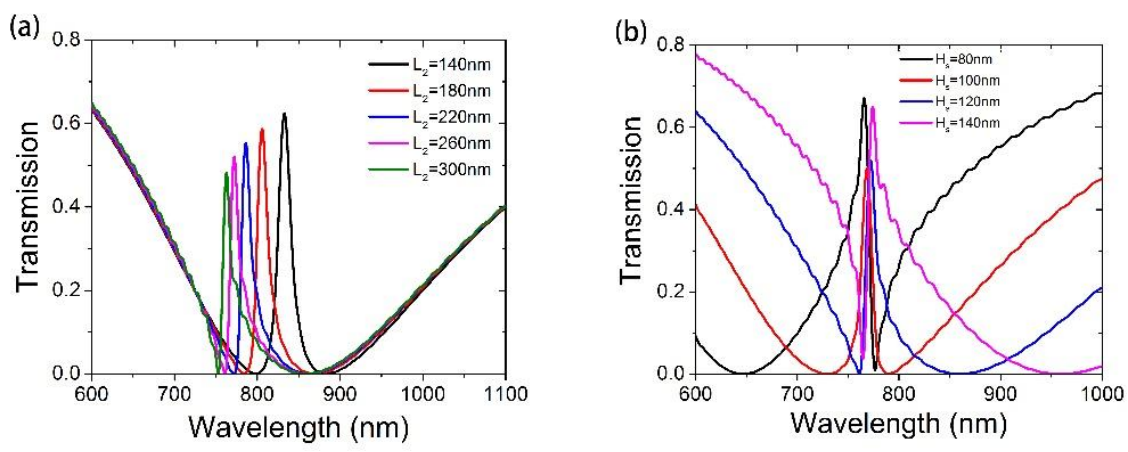

Fig. 6. (a) The transmission spectra of PIT system versus the lower bottom length of trapezoid cavity. (b) The transmission spectra with different stub height. 
There is a significant slow light effect near the PIT peak wavelength. The strength of slow light effect can be described by group index which is expressed as:

$$
n_{g}=\frac{c}{v_{g}}=\frac{c}{L} \tau_{g}=\frac{c}{L} \frac{d \psi(\omega)}{d \omega}
$$

Where $v_{g}$ is the group velocity, $L$ is the length of this system which is set as $1 \mu \mathrm{m}, \tau_{g}$ is the group delay and $\psi(\omega)$ is the phase shift as a function of the frequency of incident light. The group index is determined by these two variables via Eq. (2). With the increasing of $L_{2}$, the PIT effect recedes and leads to the decreasing of phase shift. This changing will make the group index decreasing. Meanwhile, the decreasing of coupling strength between stub and trapezoid cavity means that the Q-factor becomes larger and the FWHM becomes narrower and the slope of $\psi(\omega)$ becomes sharper, which leads to the increasing of $n_{g}$. Due to the tradeoff of these two variables, the group index increases firstly, and then decreases as shown in Fig.7 (a). The maximum of group index is approximately 17 when $L_{2}=260 \mathrm{~nm}$. While Fig. 7(b) shows the group index varys with the height of stub resonator. The maximum of group index is found when $H_{s}=110 \mathrm{~nm}$.

(a)

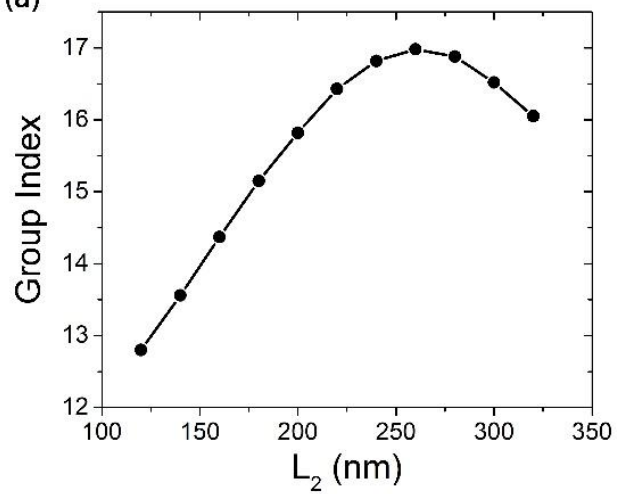

(b)

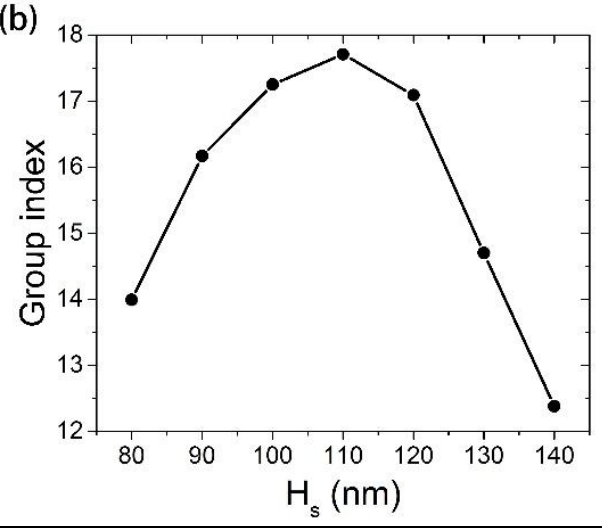

Fig. 7. Group index of the PIT system (a) versus the lower bottom length of trapezoid cavity and (b) versus the

height of stub.

\section{PIT induced by $\mathrm{TM}_{10}$ mode}

In this part, the PIT effect based on the $\mathrm{TM}_{10}$ mode was investigated and some abnormal results were obtained. The parameters of the stub are set as $W_{s}=60 \mathrm{~nm}$ and $H_{s}=100 \mathrm{~nm}$ and the resonance wavelength of stub resonator shifts to $680 \mathrm{~nm}$ which is near the resonance wavelength of $\mathrm{TM}_{10}$ mode of the trapezoid resonator. Fig. 8(a) shows the transmission spectra of stub resonator (the black line), trapezoid resonator (the red line) and the PIT system (blue line), where $L_{2}$ $=300 \mathrm{~nm}$. It is obvious that there is only one PIT peak appears at the resonance wavelength of $\mathrm{TM}_{01}$ mode. Although the resonance wavelength of $\mathrm{TM}_{10}$ mode falls into the broad stub resonance, no PIT peak appears, which is abnormal in a PIT system. Figure 8(b) and 8(c) are the $\mathrm{H}_{z}$ distributions of stub mode coupled with $\mathrm{TM}_{01}$ mode and $\mathrm{TM}_{10}$ mode of trapezoid resonator, respectively. According in Eq. (1), the coupling coefficient between the stub mode and trapezoid resonator mode is the main factor to affect the PIT effect. However, in the $\mathrm{TM}_{10}$ mode, the field strength near the center of the lower bottom is almost zero according to Fig. 2(c), so the overlap of 
stub mode and $\mathrm{TM}_{10}$ mode of trapezoid cavity is fairly small and the PIT effect is too weak to be observed.
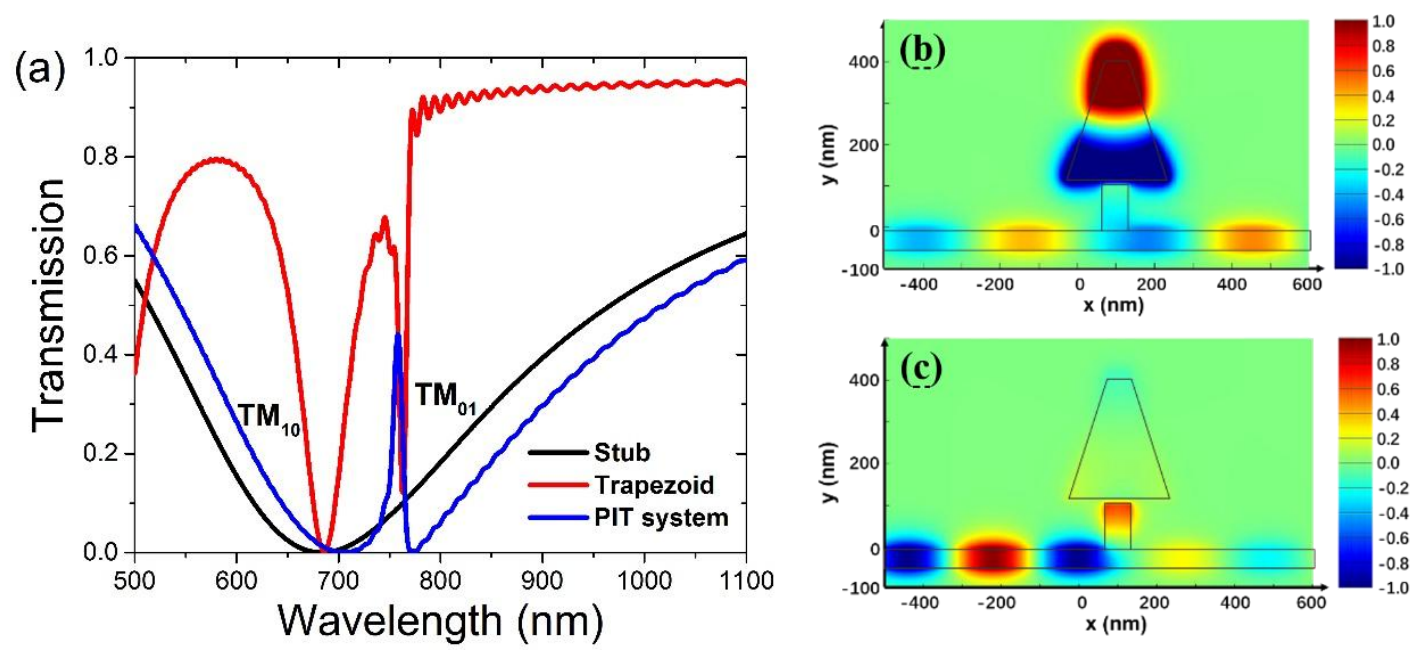

Fig. 8. (a)The transmission spectra of stub resonator, trapezoid resonator and the PIT system when $L_{2}=300$. (b)

Normalized $\mathrm{z}$ component of magnetic field amplitude of PIT systems induced by $\mathrm{TM}_{01}$ mode and (c) by $\mathrm{TM}_{10}$ mode.

To enhance the PIT effect and slow light effect, the coupling coefficient should be enlarged by increasing the field overlap between the stub mode and the $\mathrm{TM}_{10}$ mode of trapezoid. This can be realized by altering the relative position between the stub and the trapezoid resonator as shown in Fig. 9. Here, a new parameter $s$ is defined to express the shift of stub relative to the symmetry axes of the trapezoid.

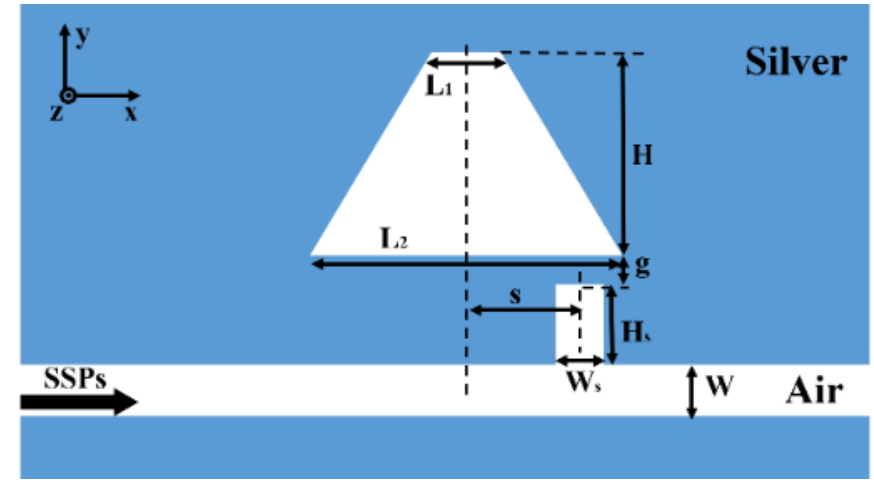

Fig.9 The new PIT system proposed to induce a PIT of $\mathrm{TM}_{10}$ mode.

Fig. 10(a) shows the transmission spectra when $\mathrm{s}=80 \mathrm{~nm}$. Here, we found the "disappeared" PIT peak reappears at the resonance wavelength of $\mathrm{TM}_{10}$ mode. The $\underline{\mathrm{H}}_{\underline{z}}$ field distributions are presented in Fig.10 (b) and Fig. 10(c). The coupling strength between stub resonator and trapezoid resonator is obviously enhanced, because the field strength is much larger at the left or right corner than at the center of the trapezoid's lower bottom. As the increasing of $s$, the PIT peak transmission increases as shown in Figure 10(d). The coupling coefficient has been enlarged dramatically because of a significant increasing of field overlap between the stub and the trapezoid cavity by altering the position of stub resonator. And it also results in a remarkable slow light effect shown in Fig. 10(e). The group index increases firstly and then decreases when $s$ increases. 
This phenomenon can be attributed to the changing near field coupling strength. When $s$ increases, the coupling strength between stub and trapezoid increases, which enhances the strength of the PIT effect. So the slow light effect is enhanced. However, as $s$ increases further, the increasing coupling strength will also reduce the Q-factor of the PIT system. Then, the FWHM of the PIT peak is broadened, that makes the slope of phase gentle [3]. Because of this tradeoff, the group index of $\mathrm{TM}_{10}$ mode reaches a maximum value about 18 when $s=30 \mathrm{~nm}$.
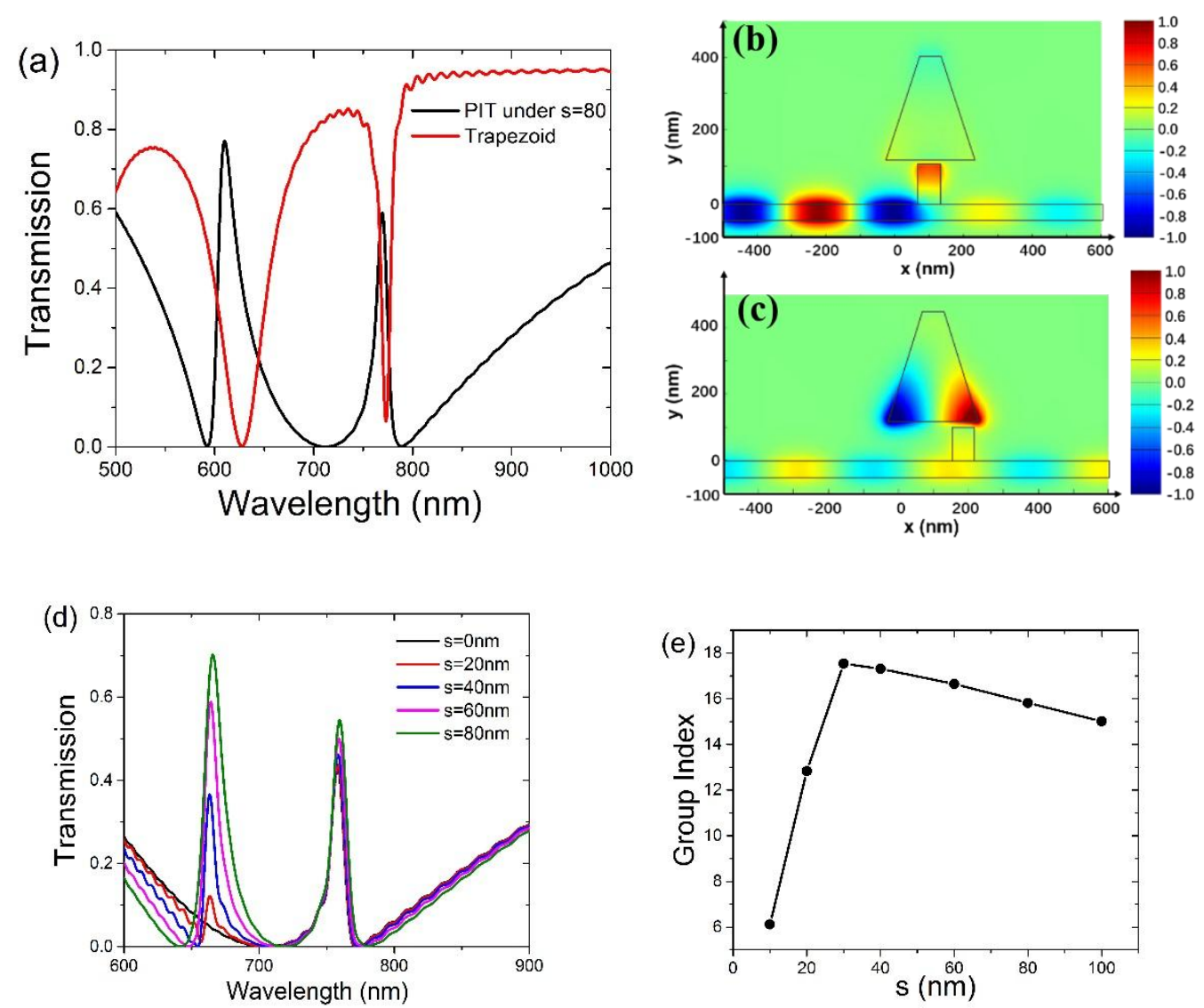

Fig. 10. (a) Transmission spectra of the PIT system when $s$ is set as $80 \mathrm{~nm}$. (b) Normalized z component of magnetic field of the PIT system without a shift and (c) with a shift of $80 \mathrm{~nm}$ at the incident wavelength of $608.86 \mathrm{~nm}$, which is the resonance wavelength of $\mathrm{TM}_{10}$ mode. (d) Transmission spectra of the PIT system when $s$ is set as $0 \mathrm{~nm}, 20 \mathrm{~nm}, 40 \mathrm{~nm}, 60 \mathrm{~nm}$ and $80 \mathrm{~nm}$. (e) The dependence of group index on $s$, the maximum of group index of this system is about 18 when $s$ is set as $30 \mathrm{~nm}$.

\section{Conclusions}

In this article, the modes of trapezoid resonator have been analyzed and a new structure, based on trapezoid cavity and stub resonator, to realize the PIT effect has been proposed and discussed. We have found that the $\mathrm{TM}_{01}$ and $\mathrm{TM}_{10}$ modes have different behaviors when they are coupled with the stub resonance to produce the PIT effect. The $\mathrm{TM}_{01}$ can bring the PIT effect effectively but no PIT effect were observed for the $\mathrm{TM}_{10}$ mode when the stub was aligned to the center of the lower bottom length of trapezoid cavity. By shifting the stub resonator to the lower corner of the trapezoid cavity, the PIT effect induced by the $\mathrm{TM}_{10}$ can be produced. The simulation results indicated that the PIT effect not only depends on the overlap between the two coupled resonant modes, but is also related to the overlap of their near fields. The observations give a 
useful supplement to the PIT effect in integrated plasmonic devices and we expect that our work would do some help for the further research of plasmonic circuits.

\section{Acknowledgment:}

This work was supported by the National Science Foundation of China under Grant No.11374048 and the Fundamental Research Funds for the Central Universities. 


\section{Reference:}

[1]. Robert W. Boyd and Daniel J. Gauthier, Transparency on an optical chip. Nature 441 (2006) 701.

[2]. Michael Fleischhauer, Atac Imamoglu, Jonathan P, Electromagnetically induced transparency: Optics in coherent media. Rev. Mod. Phys 77 (2005) 663.

[3]. Kouki Totsuka, Norihiko Kobayashi Makoto Tomita, Slow Light in Coupled-Resonator-Induced Transparency. Phys.Rev.Lett 98, (2007) 213904.

[4]. H. Lu, X. Liu, and D. Mao, Plasmonic analog of electromagnetically induced transparency in multi-nanoresonator-coupled waveguide systems. Phys.Rev.A 85 (2012) 053803.

[5]. G. Lai, R. Liang, Y. Zhang, Z. Bian, L. Yi, G. Zhan, and R. Zhao, Double plasmonic nanodisks design for electromagnetically induced transparency and slow light. Opt.Express 23 (2015) 6554.

[6]. X. Han, T. Wang, X. Li, B. Liu, and Y. He, Dynamically tunable slow light based on plasmon induced transparency in disk resonators coupled MDM waveguide system. J. Phys. D: Appl. Phys. 48 (2015) 235102

[7]. S. Zhan, H. Li, G. Cao, Z. He, B. Li, and H. Xu, Theoretical Analysis of Plasmon-Induced Transparency in Ring-resonators Coupled Channel Drop Filter Systems. Plasmonics 9 (2014) 1431.

[8]. T. Wang, Y. Zhang, Z. Hong, and Z. Han, Analogue of electromagnetically induced transparency in integrated plasmonics with radiative and subradiant resonators. Opt.Express 22 (2014) 21529.

[9]. Han, Z. and S.I. Bozhevolnyi, Plasmon-induced transparency with detuned ultracompact Fabry-Perot resonators in integrated plasmonic devices. Opt.Express 19, (2011) 3251.

[10]. X. Han, T. Wang, B. Liu, Y. He, and Y. Zhu, Tunable Triple Plasmon-Induced Transparencies in Dual T-Shaped Cavities Side-Coupled Waveguide. IEEE Photonics Technology Letters 28 (2016) 347.

[11]. D. Liu, Y. Sun, Q. Fan, M. Mei, J. Wang, Y. Pan, and J. Lu, Tunable Plasmonically Induced Transparency with Asymmetric Multi-rectangle Resonators. Plasmonics, (2016) DOI 10.1007/s11468-016-0218-1.

[12]. G. Cao, H. Li, Y. Deng, S. Zhan, Z. He, and B. Li, Plasmon-induced transparency in a single multimode stub resonator. Opt.Express 22 (2014) 25215.

[13]. B. X. Li, H. J. Li, L. L. Zeng, S. P. Zhan, Z. H. He, Z. Q. Chen, and H. Xu, High-Sensitivity Sensing Based on Plasmon-Induced Transparency. IEEE Photonics Journal 7 (2015) 4801207.

[14]. K. Wen, L. Yan, M. Wei Pan, B. Luo, Z. Guo, Y. Guo, and X. Luo, Electromagnetically induced transparency-like transmission in a compact side-coupled T-shaped resonator. Journal of Lightwave Technology $\underline{32(2014) 1701 .}$

[15]. K. Wen, Y. Hu, L. Chen, J. Zhou, M. He, L. Lei, and Z. Meng, Multiple Plasmon-Induced Transparency Responses in a Subwavelength Inclined Ring Resonators System. IEEE Photonics Journal, 7 (2015) 1.

[16]. C. Song, S. Qu, J. Wang, B. Tang, and X. Xia, Plasmonic tunable filter based on trapezoid resonator waveguide. Journal of Modern Optics 64 (2015) 1400.

[17].D. F. P. Pile, T. Ogawa, D. K. Gramotnev, Y. Matsuzaki, K. C. Vernon, K. Yamaguchi, T. Okamoto, M. Haraguchi and M. Fukui, Two-dimensionally localized modes of a nanoscale gap plasmon waveguide. Appl. Phys. Lett. 87 (2005) 261114.

[18]. C. Haffner, W. Heni, Y. Fedoryshyn, J. Niegemann, A. Melikyan, D. L. Elder, B. Baeuerle, Y. Salamin, A. Josten, U. Koch, C. Hoessbacher, F. Ducry, L. Juchli, A. Emboras, D. Hillerkuss, M. Kohl, L. R. Dalton, C. $\underline{\text { Hafner and J. Leuthold, All-plasmonic Mach-Zehnder modulator enabling optical high-speed communication at }}$ the microscale, Nature Photon. 9 (2015) 525

[19]. H. U. Yang, J. D, Archangel, M. L. Sundheimer, E. Tucker, and G. D. Boreman, Optical dielectric function of silver, Phys. Rev. B 91 (2015) 235137.

[20]. Junghyun Park, Hwi Kim, and Byoungho Lee, High order plasmonic Bragg reflection in the 
metal-insulator-metal waveguide Bragg grating, Opt. Express 16 (2008) 413.

[21]. K. Wen, Y. Hu, L. Chen, J. Zhou, L. Lei, and Z. Guo, Z, Fano resonance with ultra-high figure of merits based on plasmonic metal-insulator-metal waveguide. Plasmonics, 10 (2015) 27.

[22]. K. Wen, Y. Hu, L. Chen, J. Zhou, L. Lei, and Z. Meng, Single/dual Fano resonance based on plasmonic metal-dielectric-metal waveguide. Plasmonics, 11 (2016) 315. 